Article

\title{
Use of Silver Nanoparticle-Gelatin/Alginate Scaffold to Repair Skull Defects
}

\author{
Yang Zhao, Jun Liu, Mingyue Zhang, Jia He, Bowen Zheng, Fan Liu, Zhenjin Zhao * and Yi Liu * \\ Department of Orthodontics, School of Stomatology, China Medical University, Liaoning Provincial Key \\ Laboratory of Oral Diseases, Shenyang 110001, China; yzhao79@cmu.edu.cn (Y.Z.); jliu89@cmu.edu.cn (J.L.); \\ myzhang97@cmu.edu.cn (M.Z.); jiahe@cmu.edu.cn (J.H.); bwzheng@cmu.edu.cn (B.Z.); fanliu@cmu.edu.cn (F.L.) \\ * Correspondence: zjzhao@cmu.edu.cn (Z.Z.); liuyi@cmu.edu.cn (Y.L.); Tel.: +86-24-3192-7709 (Z.Z.); \\ +86-24-3197-3999 (Y.L.)
}

Received: 22 August 2020; Accepted: 24 September 2020; Published: 30 September 2020

\begin{abstract}
The major objectives of this study were to investigate the effects of silver nanoparticlesgelatin (AgNPs) on the physical and chemical properties of gelatin/alginate (Gel/Alg) scaffolds and the bone-promoting effect of AgNP-Gel/Alg scaffolds. Gel/Alg scaffolds consisting of $0 \mu \mathrm{M}, 200 \mu \mathrm{M}$, $400 \mu \mathrm{M}$, and $600 \mu \mathrm{M}$ AgNPs were prepared. SEM was used to evaluate the physical properties of the scaffolds. A CCK-8 assay was performed to determine the cell proliferation activity, and Micro-CT and histological analysis were used to assess the osteogenic effect. The pore size, porosity, and the water absorption and degradation rates of AgNP-Gel/Alg scaffolds were found to be increased compared with those of Gel/Alg scaffolds (control group). CCK-8 showed that cell proliferation activity in the $200 \mu \mathrm{M}$ group was significantly higher than that in the control group. Micro-CT analysis showed that there was more new bone around AgNP-Gel/Alg than the control group, and the amount of bone formation in the $200 \mu \mathrm{M}$ group was significantly higher than that in the other groups. Masson staining showed that numerous collagen fibers had proliferated around the AgNP-Gel/Alg scaffold and tended to thicken over time. AgNP-Gel/Alg scaffolds promoted the repair of skull defects in New Zealand rabbits and exerted a marked osteogenic effect in vivo. The $200 \mu \mathrm{M}$ AgNP-Gel/Alg scaffold was shown to be more suitable for bone tissue engineering materials.
\end{abstract}

Keywords: Gel/Alg; AgNPs; bone repair

\section{Introduction}

In clinical practice, jaw defects or alveolar resorption caused by cysts of the jaw, tumors, trauma, and periodontal and endodontic disease are very common [1]. For a long time, autogenous bone has been defined as the gold standard for bone repair material owing to its excellent osteoconduction, osteogenesis, and osteoinduction in replacing damaged or missing bones. However, secondary surgery of autogenous bone increases the pain and risk for patients [2-4].

In recent years, with the in-depth understanding, development and utilization of bone tissue engineering material, biomaterial has gradually become a new substitute for bone tissue, opening a new path for the treatment of related diseases. Because it naturally draws in material, induces a low- level immune response, and has great biocompatibility, hydrophilicity, and biodegradability, gelatin (Gel) has attracted wide attention [5]; however, it also has demerits such as fast degradation and poor mechanical properties, and the pore diameter of a Gel scaffold is large. As a natural polysaccharide, alginate (Alg) can form scaffolds in a relatively short time and adjust the pore diameter of the scaffold to make up for the defects of Gel [6]. Therefore, Gel/Alg scaffolds are widely used [7].

Silver nanoparticles-gelatin (AgNPs) not only have broad-spectrum antibacterial activity and are effective against antibiotic-resistant strains but can also promote the proliferation and migration 
of keratinocytes [8]. Because of their remarkable antibacterial effect, AgNPs have been widely used in the medical field, especially in implants of bone transplantation [9]. In addition, previous studies have focused on the cytotoxicity and genotoxicity of AgNPs, but little is known about its effects on cell differentiation [10]. Other studies have shown that AgNPs can promote fibroblasts to differentiate into myofibroblasts and accelerate wound contraction [11,12]. Hui Q et al. [13] found that AgNPs at appropriate concentrations can promote the osteogenic differentiation of urine-derived stem cells. Zhang R et al. [14] found that AgNPs can promote osteogenic differentiation of mammalian bone marrow mesenchymal stem cells. In the early stage of the study, AgNPs were shown to improve the osteogenic differentiation of periodontal ligament fibroblasts [15]. However, the ability of AgNPs to promote osteogenesis in vivo has not been reported.

The aim of our study was to evaluate the osteogenic effect of AgNPs in vivo by preparing AgNP-Gel/Alg scaffolds and applying them to defect models of the New Zealand rabbit skull.

\section{Materials and Methods}

\subsection{Preparation of AgNP-Gel/Alg Scaffolds}

Scaffolds containing AgNPs were initially prepared. First, $0.015 \mathrm{~g}$ of Alg and $2 \mathrm{~g}$ of Gel powder were added to $10 \mathrm{~mL}$ of deionized water, and the mixture was stirred rapidly at high temperature in a magnetic stirring apparatus until dissolved to obtain $0.15 \%(w / v)$ Alg and 20\% (w/v) Gel solutions. $\mathrm{Alg}$ and Gel were mixed in equal volumes and added to the AgNP solution, and then the mixture was subjected to ultrasound treatment to obtain liquids containing Gel/Alg mixed with different concentrations of AgNPs, namely, $0 \mu \mathrm{M}, 200 \mu \mathrm{M}, 400 \mu \mathrm{M}$, and $600 \mu \mathrm{M}$. The evenly mixed solution was poured into a 48 -well plate, stored at $-20^{\circ} \mathrm{C}$ overnight and lyophilized for $48 \mathrm{~h}$. The cross-linked scaffold was incubated in $2 \% \mathrm{CaCl}_{2}$ overnight at $4{ }^{\circ} \mathrm{C}$ and cleaned in distilled water to remove additional $\mathrm{CaCl}_{2}$. Then, the cross-linked scaffold was placed in a $2 \%$ glutaraldehyde solution for $30 \mathrm{~min}$ and rinsed with distilled water to remove the additional glutaraldehyde. The cross-linked scaffold was stored overnight at $-20^{\circ} \mathrm{C}$ and lyophilized at $-50^{\circ} \mathrm{C}$ for $48 \mathrm{~h}$.

\subsection{Morphological Characterization of the AgNP-Gel/Alg Scaffold}

The morphology of AgNP-Gel/Alg was determined by a scanning electron microscope (SEM, CamScan MX2600FE, West Sussex, UK). A scaffold slice, which was obtained with a scalpel, was fixed to conductive glue, and the surface of the sample was iron-sputtered. SEM images were collected at an acceleration voltage of $15 \mathrm{kV}$ and 200 times magnification. The pore diameter of the prepared sample was analyzed and detected by Image J analysis software.

\subsection{Determination of the Porosity of the Scaffold}

First, the weight of the scaffold sample (diameter: $10 \mathrm{~mm}$; thickness: $3 \mathrm{~mm}$ ) was measured and recorded as W1. Liquid displacement was used to measure the scaffold porosity. Because ethanol has no effect on the expansion or contraction of the scaffold, it was chosen as the replacement fluid. The geometrical volume $(\mathrm{V})$ of the cylindrical scaffold was calculated, and the scaffold was submerged in pure ethanol in a vacuum for $20 \mathrm{~min}$. In order to induce the ethanol to infiltrate the scaffold, it was continuously circulated in a vacuum pumping and recompression system until there were no bubbles. After removing excess ethanol from the surface with filter paper, the weight of the scaffold was determined and recorded as W2. Porosity was calculated by the following equation:

$$
\text { Porosity }(\%)=(\mathrm{W} 2-\mathrm{W} 1) / \rho \mathrm{V} \times 100\left(\rho=0.789 \mathrm{mg} \cdot \mathrm{mL}^{-1}\right)
$$

\subsection{Determination of the Swelling Ratio of the Scaffold}

First, the weight of the scaffold sample (diameter: $10 \mathrm{~mm}$; thickness: $3 \mathrm{~mm}$ ) was determined and recorded as W1. The scaffold was soaked in $1 \times \mathrm{PBS}$ at $37^{\circ} \mathrm{C}$ for $24 \mathrm{~h}$ to measure its water absorption. 
At the end of the swelling phase, the scaffold was cleaned in deionized water to remove any ions absorbed on the surface and then dried with filtered water. The wet weight was recorded as W2. The rate of swelling of the scaffold was calculated using the following formula: swelling rate $(\%)=$ $(\mathrm{W} 2-\mathrm{W} 1) / \mathrm{W} 1 \times 100$.

\subsection{Determination of the Degradation Rate of the Scaffold}

In vitro biodegradability of the scaffold was tested by incubating the scaffold in the PBS solution and evaluating the weight loss of the samples. The scaffold was cut into samples with the same weight (W1) and soaked in $25 \mathrm{~mL}$ of PBS ( $\mathrm{pH}=7.4)$, before which the scaffold was sterilized for about $15 \mathrm{~min}$ with 70\% ethanol and washed three times with PBS. Incubation was performed in the dark. The samples were incubated at $37^{\circ} \mathrm{C}$ in PBS for 2, 4, and 6 weeks, and then the remaining scaffold was separated, washed thoroughly with deionized water, and dried in a vacuum at $50{ }^{\circ} \mathrm{C}$ until it reached a constant weight (W2). The weight loss due to deterioration of the scaffold was calculated using the equation. Each scaffold was measured at least three times, and the average value was recorded. The rate of degradation was calculated using the following formula: Degradation rate $(\%)=(\mathrm{W} 1-\mathrm{W} 2) / \mathrm{W} 1 \times 100$.

\subsection{Cell Proliferation in the Scaffold}

MC3T3-E1 cells were cultivated in MEM medium containing 10\% fetal bovine serum (FBS), $100 \mathrm{U} / \mathrm{mL}$ penicillin, and $100 \mu \mathrm{g} / \mathrm{mL}$ streptomycin in a humidified incubator at $37{ }^{\circ} \mathrm{C}$ and $5 \% \mathrm{CO}_{2}$. After reaching 80-90\% fusion, the fifth generation of cells was centrifuged to produce a cell suspension with a cell density of $1 \times 10^{5} / \mathrm{mL}$. Cell suspensions $(50 \mathrm{uL})$ were carefully inoculated on the surfaces of $0 \mu \mathrm{M}, 200 \mu \mathrm{M}, 400 \mu \mathrm{M}$, and $600 \mu \mathrm{M}$ AgNP-Gel/Alg scaffolds (diameter: $10 \mathrm{~mm}$; thickness: $3 \mathrm{~mm}$ ) with an inoculation density of 5000 cells/wells. Each group was set in three wells as replicates and placed in a $37^{\circ} \mathrm{C}$ incubator for cell adhesion. After $4 \mathrm{~h}, 500 \mathrm{uL}$ of the medium was added. After incubation for 1 , 3,5 , and 7 days, $300 \mathrm{uL}$ of the medium was replaced with fresh medium, and $30 \mathrm{uL}$ of cholecystokinin- 8 (CCK-8) reagent was added to every well and thoroughly mixed. The culture plate was placed in an incubator for $2 \mathrm{~h}$. After that, $100 \mu \mathrm{L}$ of the medium was transferred to a 96-well plate, and the OD value was measured at $450 \mathrm{~nm}$.

\subsection{Model Preparation of Critical Skull Defects of Rabbits and Animal Grouping}

The protocol was approved by the Ethical Committee and the Laboratory Animal Center of China medical university, Shenyang, Liaoning, People's Republic of China. New Zealand white rabbits were weighed and intramuscularly injected with $0.2 \mathrm{~mL}$ of new chloramine ketone solution. The hair over the rabbit's ear vein was removed with a shaver, and $3 \%(1 \mathrm{~mL} / \mathrm{kg})$ sodium pentobarbital solution was injected into the ear vein. The rabbit underwent local anesthesia with primacaine adrenaline. A longitudinal incision of about $5 \mathrm{~cm}$ was cut along the midline of the skull; the skin and mucoperiosteum were peeled off with a dissector, and the operation area was completely exposed. To avoid damaging the dura, four circular full-thickness bone defects with a diameter of $10 \mathrm{~mm}$ were drilled along both sides of the suture with a ring drill, during which ice brine was used for physical cooling. The operation area was locally injected with $1 \mathrm{~mL}$ of 400,000 units of the scaffold material in penicillin sodium solution and sutured. An intramuscular injection of 400,000 units of penicillin sodium solution was administered for three consecutive days after the operation.

Twelve New Zealand white rabbits were divided randomly into two groups based on the time of observation. Four scaffolds (Gel/Alg (control group), $200 \mu \mathrm{M}$ AgNP-Gel/Alg, $400 \mu \mathrm{M}$ AgNP-Gel/Alg, and $600 \mu \mathrm{M}$ AgNP-Gel/Alg) were implanted at random into the skull defects of rabbits, and the wounds were closed with stratified suture. After the operation, the rabbits were placed in cleaning cages, and wound healing and inflammatory reactions were observed after they woke up (Figure 1). 


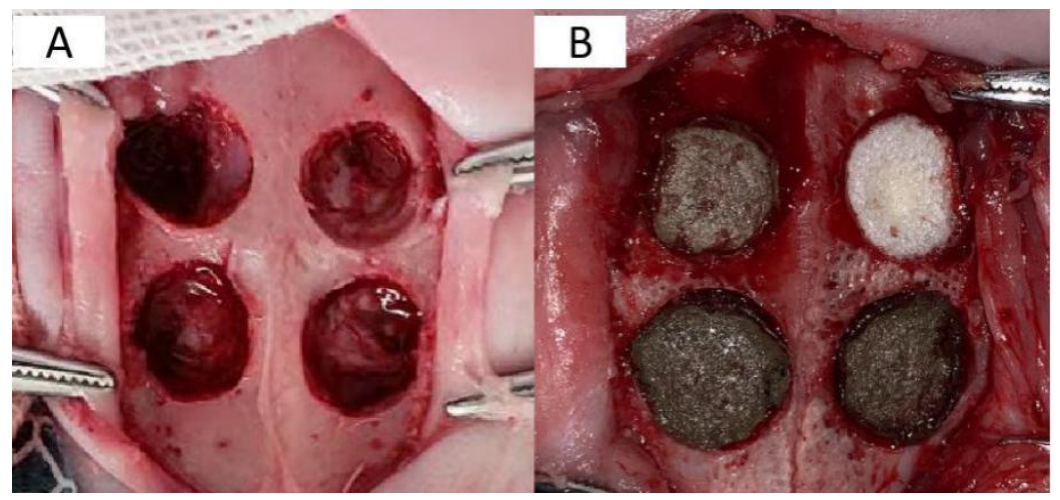

Figure 1. Rabbit skull defect preparation and material implantation: (A). reparation of rabbit skull defect models; (B). the implantation of AgNP-Gel/Alg stents with different scaffolds.

\subsection{Iconographical and Histological Analyses after the Operation}

After surgery, six animals were sacrificed at 4 and 8 weeks. The skull defect locations were exposed, and peripheral bones and soft tissues were surgically removed. The critical skull bone defect sites were scanned by micro-computed tomography (Micro-CT, Inspexio; Shimadzu Corporation, Tokyo, Japan) for X-ray imaging. Three-dimensional reconstruction was performed using TRI/3DBON software (RATOC Systems Engineering, Tokyo, Japan) from a set of acquired scans.

Iconographic assessment was carried out by Micro-CT of critical bone defect sites in animals sacrificed at 4 and 8 weeks after surgery. Tissue blocks were removed and fixed with $4 \%$ paraformaldehyde solution for $72 \mathrm{~h}$, and step-by-step dehydration from low to high concentrations (70\%, $80 \%, 90 \%$ and $100 \%)$ was performed, followed by xylene clearing, plastic dipping and resin embedding; then, $5-8 \mu \mathrm{m}$ slices were obtained by microtome and de-plasticized in water, followed by Masson staining, clearing and finally sealing. Three slices were cut from each tissue sample, and two slices were selected for observation for different visualizations under the microscope.

\subsection{Statistical Analysis}

The experimental results are shown as means \pm standard deviation (SD). The data were processed with a one-way variance analysis (ANOVA) by SPSS 22.0 software (SPSS, Chicago, IL, USA), and GraphPad Prism 8.0 software was used for drawing. Differences are considered to be statistically significant at $p<0.05$.

\section{Results}

\subsection{Microstructure and General Observation of Scaffolds}

The scaffold material was cylindrical and had pinhole-sized pores visible to the naked eye, with a height of $3 \mathrm{~mm}$ and a base diameter of $10 \mathrm{~mm}$. SEM showed that the microstructures of the four materials were highly porous and interconnected (Figure 2). There was an excellent connection between holes, which could ensure the implantation of cells and the transport of nutrients. The control group and $200 \mu \mathrm{M}$ AgNP-Gel/Alg scaffolds had smooth edges, abundant pores, and good pore-forming effects. With the increase in AgNP concentration, the pore morphology of the scaffold contained more flaky sharp edges.

\subsection{Determination of the Porosity and Pore Diameter of Scaffolds}

With the increase in AgNP concentration, the pore diameter and porosity of the scaffolds increased (Figure 3). The pore size and porosity of the experimental groups with different concentrations of AgNPs were significantly increased compared with those of the control group. In the AgNP 
groups, there was no substantial difference in pore size or porosity between the $400 \mu \mathrm{M}$ and $600 \mu \mathrm{M}$ AgNP-Gel/Alg groups.

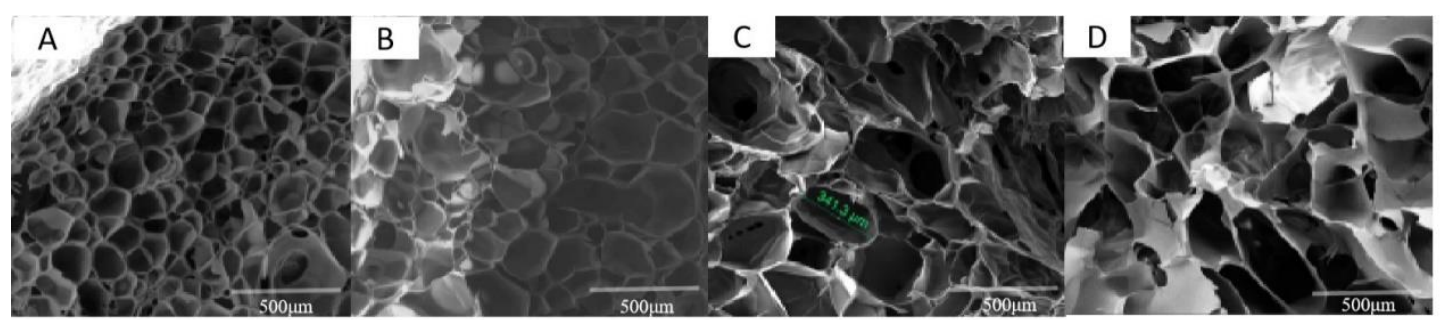

Figure 2. Scanning electron microscopy (SEM) images of AgNP-Gel/Alg scaffolds: (A). Gel/Alg scaffold (control group); (B). $200 \mu \mathrm{M}$ AgNP-Gel/Alg scaffold; (C). $400 \mu \mathrm{M}$ AgNP-Gel/Alg scaffold; (D). $600 \mu \mathrm{M}$ AgNP-Gel/Alg scaffold.
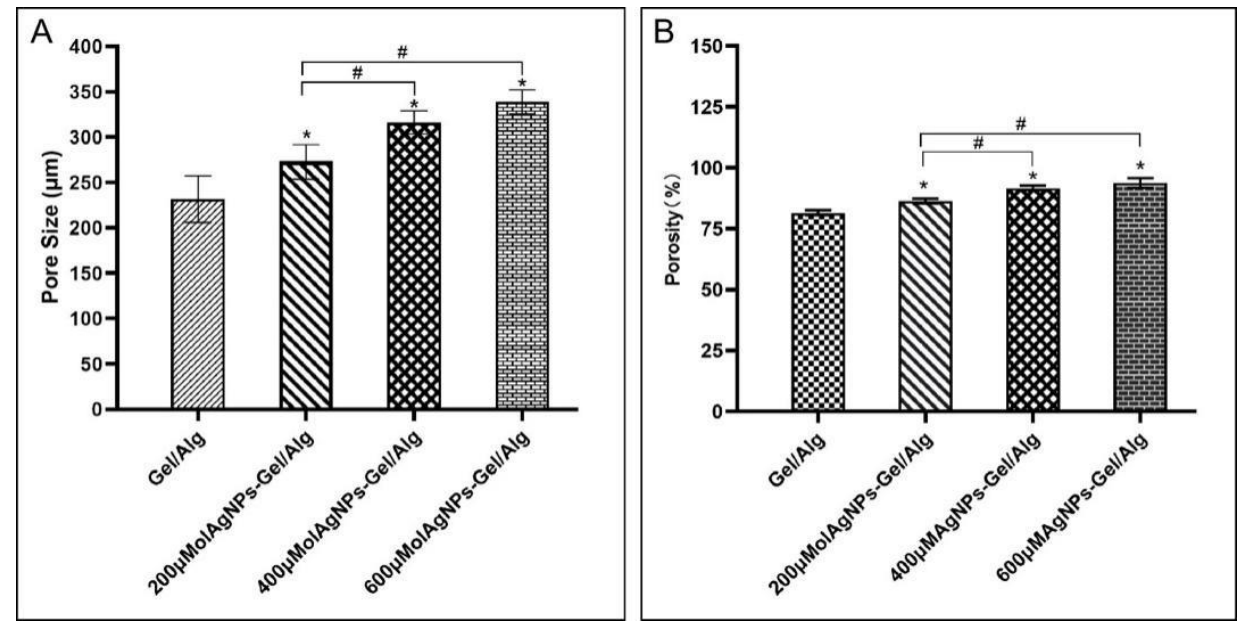

Figure 3. Pore diameter and porosity of the Gel/Alg scaffold after adding different concentrations of AgNPs: (A). pore diameter; (B). porosity. ${ }^{*} p<0.05$ vs. control group; ${ }^{\#} p<0.05$ vs. $200 \mu \mathrm{M}$ AgNP-Gel/Alg group.

\subsection{The Scaffold Swelling Rate}

The swelling rate was substantially higher for the AgNP groups than the control group. In the AgNP group, there was no significant difference in the swelling rate between the $400 \mu \mathrm{M}$ and $600 \mu \mathrm{M}$ AgNP-Gel/Alg groups $(p>0.05)$ (Figure 4).

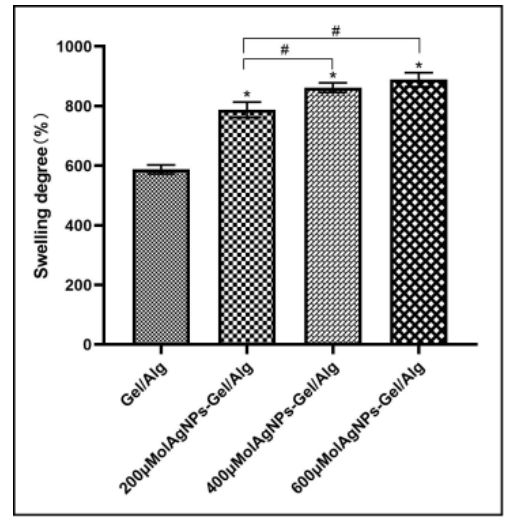

Figure 4. Swelling rate of AgNP-Gel/Alg scaffold with different concentrations of AgNPs. ${ }^{*} p<0.05$ vs. control group; ${ }^{\#} p<0.05$ vs. $200 \mu \mathrm{M}$ AgNP-Gel/Alg group. 


\subsection{Degradation Rate of Scaffolds}

With the increase in AgNP concentration, the degradation rate of the AgNP groups increased. In the AgNP groups, there was no significant difference in the degradation rate between the $400 \mu \mathrm{M}$ and $600 \mu \mathrm{M}$ AgNP-Gel/Alg groups. With the increase in time, the degradation rate of each group increased (Figure 5).

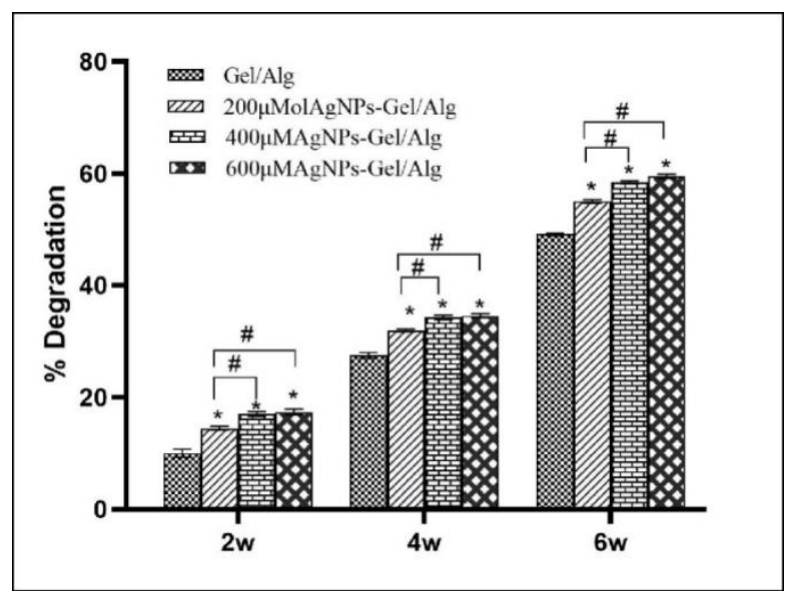

Figure 5. In vitro degradation rate of AgNP-Gel/Alg scaffold with different concentrations of AgNPs. ${ }^{*} p<0.05$ vs. control group; ${ }^{\#} p<0.05$ vs. the $200 \mu \mathrm{M}$ AgNP-Gel/Alg group.

\subsection{Cell Proliferation on Scaffolds}

MC3T3-E1 cell proliferation in the AgNP groups and control group after 1, 3, 5, and 7 days is shown in Figure 6. On day 1, the cell proliferation activities in the AgNP groups and the control group were not significantly different. On day 3, all the MC3T3-E1 cells in the scaffold entered a phase of rapid proliferation, and the cell proliferation activity of the $200 \mu \mathrm{M}$ AgNP-Gel/Alg group was significantly higher than that of the control group $(p<0.05)$. On day 5 , the cell proliferation activity of the 600 $\mu \mathrm{M}$ AgNP-Gel/Alg group was not significantly different from that of the control group $(p<0.05)$. In groups of AgNPs, in the $400 \mu \mathrm{M}$ and $600 \mu \mathrm{M}$ AgNP-Gel/Alg groups, there was no significant difference in cell proliferation activity. On day 7 , the cell proliferation activity of scaffolds with different concentrations of AgNPs was significantly higher than that of the control group $(p<0.05)$. The cell proliferation activity of the $200 \mu \mathrm{M}$ AgNP-Gel/Alg group was significantly higher than that of the $600 \mu \mathrm{M}$ AgNP-Gel/Alg group $(p<0.05)$ (Figure 6).

\subsection{Micro-CT Imaging Analysis}

Figure 7 shows representative observations from Micro-CT imaging: at 4 and 8 weeks, the control group was found to have less new bone formation. In the AgNP groups, more new bone tissue was found at the edge of primary bone tissue. At 4 weeks, the new bone volume (BV) of the 200 and $400 \mu \mathrm{M}$ AgNP-Gel/Alg groups was substantially higher than that of the control group $(p<0.05)$. There were substantial differences in BV among the AgNP groups $(p<0.05)$ (Figure 8A). With the increase in time, the BV value of each group increased.

At 4 weeks, the trabecular bone connectivity (Tb.Pf) of the $200 \mu \mathrm{M} \mathrm{AgNP-Gel/Alg} \mathrm{group} \mathrm{was}$ less than that of the control group $(p<0.05)$. The Tb.Pf of the $400 \mu \mathrm{M}$ and $600 \mu \mathrm{M} \mathrm{AgNP-Gel/Alg}$ groups was greater than that of the $200 \mu \mathrm{M}$ AgNP-Gel/Alg group (Figure 8B). With the increase in time, the Tb.Pf values of each group decreased. 


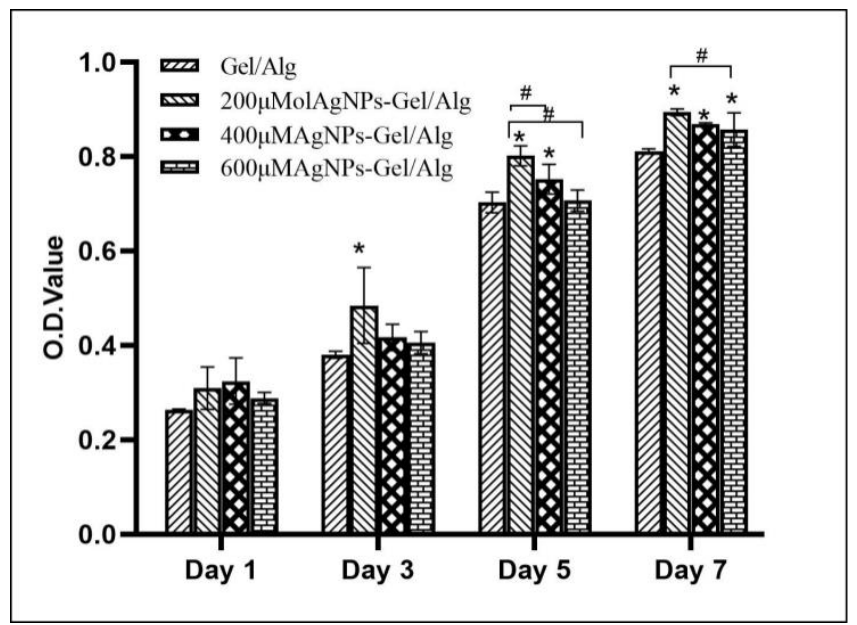

Figure 6. Proliferation of MC3T3-E1 cells on AgNP-Gel/Alg scaffolds with different concentrations of AgNPs. ${ }^{*} p<0.05$ vs. control group; ${ }^{\#} p<0.05$ vs. $200 \mu \mathrm{M}$ AgNP-Gel/Alg group.

A

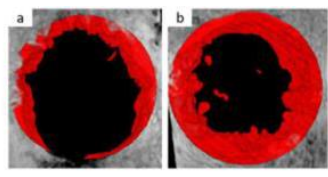

B

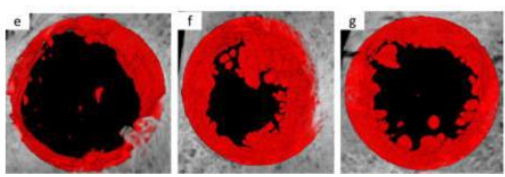

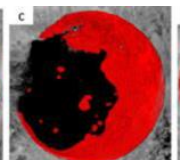
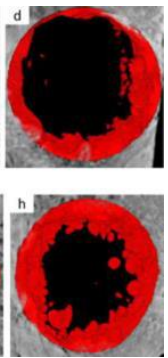

Figure 7. Micro-CT images: (A) 4 weeks after surgery; (B) 8 weeks after surgery; (a,e) Gel/Alg group; (b,f) $200 \mu \mathrm{M}$ AgNP-Gel/Alg group; (c,g) $400 \mu \mathrm{M}$ AgNP-Gel/Alg group; (d,h) $600 \mu \mathrm{M}$ AgNP-Gel/Alg group.
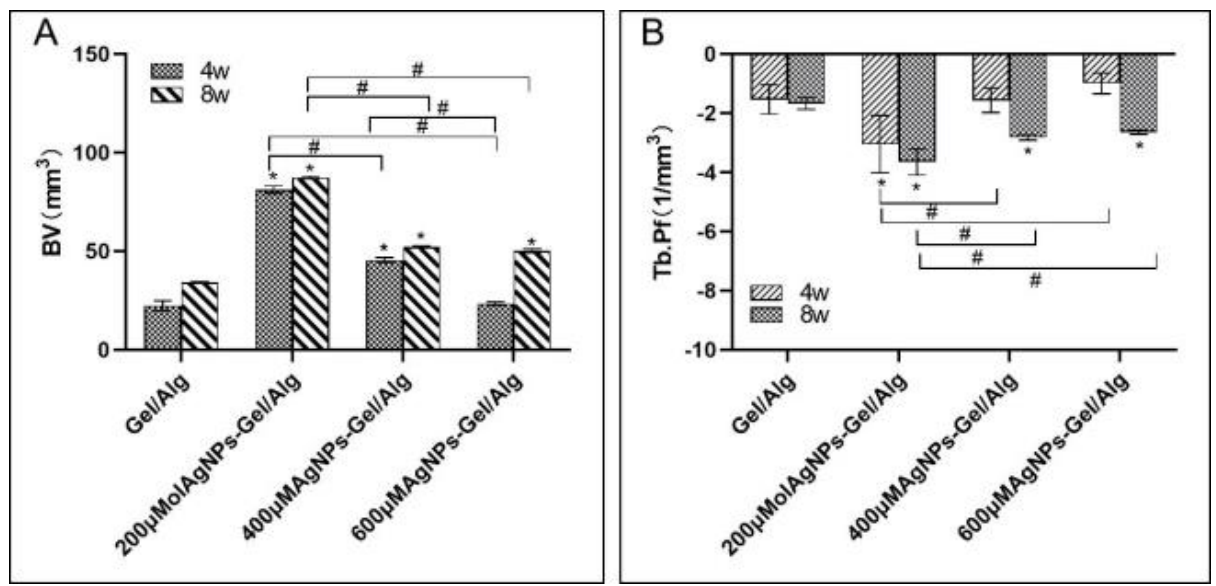

Figure 8. (A) New bone volume (BV) and (B) trabecular bone connectivity (Tb.Pf) in different groups at 4 and 8 weeks after operation. ${ }^{*} p<0.05$ vs. control group; ${ }^{\#} p<0.05$ the difference between AgNPGel/Alg groups.

\subsection{Histological Analysis}

At 4 weeks, trabecular fibrous tissue was observed in the $200 \mu \mathrm{m}$ and $400 \mu \mathrm{m} \mathrm{AgNP-Gel/Alg}$ groups. However, there was little tissue in the control group and $600 \mu \mathrm{m} \mathrm{AgNP-Gel/Alg} \mathrm{group.}$ With the increase in time, the trabecular cord-like fibrous tissue thickened and increased (Figure 9). 
A

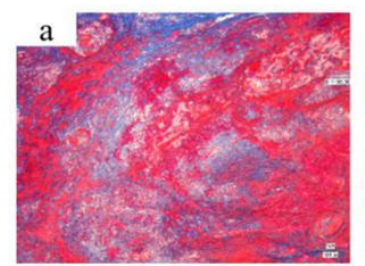

B

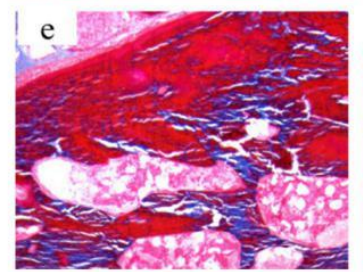

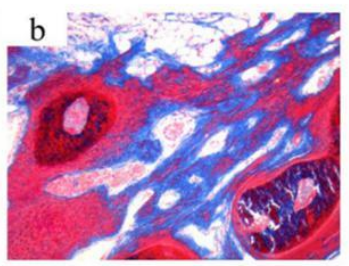

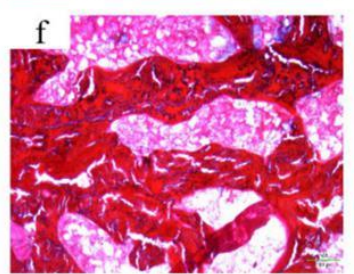

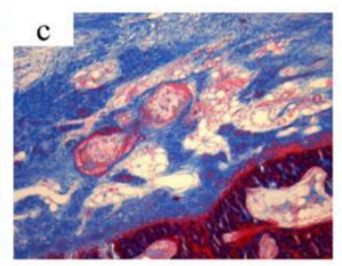

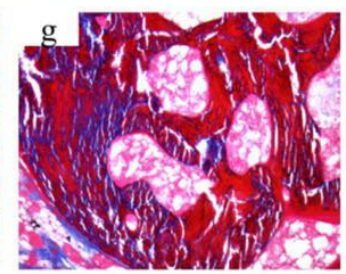

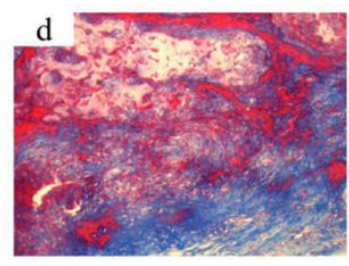

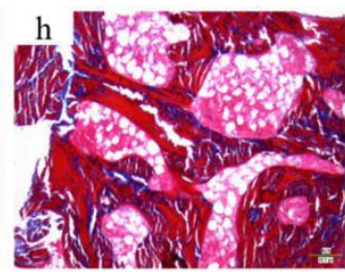

Figure 9. Masson stain (100×): (A) 4 weeks after surgery; (B) 8 weeks after surgery; (a,e) Gel/Alg group; (b,f) $200 \mu \mathrm{M}$ AgNP-Gel/Alg group; (c,g) $400 \mu \mathrm{M}$ AgNP-Gel/Alg group; (d,h) $600 \mu \mathrm{M}$ AgNP-Gel/Alg group.

\section{Discussion}

In this research, AgNP-Gel/Alg composite bone tissue engineering scaffolds were prepared using a freeze-drying technique. Because AgNPs may affect the chemical and physical properties of the scaffolds, such as pore size, porosity, in vitro enzymatic degradation, morphology, and swelling behavior, we observed the effects of AgNPs on these properties. The pore size in the scaffold structure can affect the mechanical properties of the scaffold and regulate cell adhesion, proliferation, vascularization, and osteogenic differentiation. Many researchers believe that scaffold materials with pore sizes in the range of $100-600 \mu \mathrm{M}$ are the most suitable for bone growth and should be as close as possible to the size of normal bone units (about $223 \mu \mathrm{m}$ ) [16]. The pore size of the composite scaffold prepared in this experiment is between $230 \mu \mathrm{m}$ and $350 \mu \mathrm{m}$, and the pore size increases with the increase in AgNP concentration. The reason for this result may be that the interaction between $\mathrm{Ag}+$ and $-\mathrm{NH}_{2}$ or $-\mathrm{COOH}$ in Gel leads to a decrease in the hydrogen bonding of gelatin chains, resulting in a loose connection between gelatin chains and increased pore sizes with increasing concentrations of AgNPs. The average pore size of the $200 \mu \mathrm{M}$ AgNP-Gel/Alg scaffold is $272.8 \mu \mathrm{m}$, which is close to the normal bone unit size and more in line with the requirements of bone scaffold materials.

Biomaterial scaffolds play a significant role in bone formation in vivo because of their porosity and pore size. Appropriate porosity enables porous structures to interconnect with each other and provides channels for nutrient transport, waste discharge, and "inward" growth of new tissues. Kruyt et al. studied in vivo bone formation on a hydroxyapatite (GMSC) scaffold with 70\% porosity and an average pore size of $800 \mu \mathrm{M}$ and a GMSC scaffold with $60 \%$ porosity and an average pore size of $400 \mu \mathrm{M}$. However, when the GMSC scaffold was implanted into the bilateral paraspinal muscles of goats, the $70 \%$ $800 \mu \mathrm{M}$ scaffold formed more bone. This result may be due to greater ion exchange and adsorption of osteoinductive factors because of a larger surface area [17]. Although increasing porosity and pore size can provide better pore connectivity and inward growth of bone cells, it also reduces mechanical properties. Chitosan sponges with a pore size of $100 \mu \mathrm{M}$ were formed in hydroxyapatite/ $\beta$-tricalcium phosphate scaffolds with large pores $(300-600 \mu \mathrm{m})$. The compression module and the yield stress increased by around four times [18]. The porosity of bone tissue engineering scaffolds should be above $60-80 \%$. In this study, the porosity of the composite scaffolds was above $80 \%$, and the porosity increased with the increase in AgNP concentration. All scaffold materials in this study had good absorption and swelling ability because gelatin and sodium alginate are hydrophilic natural polymer materials, and the pore size and porosity of the porous materials are conducive to water absorption.

Degradability is also an important indicator when measuring the performance of bone repair materials. In the process of cell growth and tissue formation, the biodegradation of the scaffold continues until the scaffold is completely degraded. The rate of biodegradation should follow the growth rate 
of the cells so that as the scaffold area is reduced, the cells fill the degraded area. Biodegradation of scaffolds usually occurs by hydrolysis, dissolution, transformation or enzymatic decomposition [19]. Figure 6 shows the degradation degree of AgNP-Gel/Alg after incubation in PBS solution for different times. As the AgNP content in the scaffold increased, the degradation rate increased correspondingly. In this experiment, the average degradation rate of $200 \mu \mathrm{M} \mathrm{AgNP}-\mathrm{Gel} / \mathrm{Alg}$ scaffolds was $33.8 \%$, while that of $600 \mu \mathrm{M}$ AgNP-Gel/Alg scaffolds was $37.1 \%$. This is because the degradation rate of the material is proportional to the surface area, and the larger the porosity, the faster the degradation rate. The experiment was conducted in a PBS buffer environment, and the lack of degradation enzymes resulted in a slow degradation rate, which made it difficult to maintain consistency with the situation in vivo.

Because of their excellent antibacterial properties, silver-plated orthopedic implants have attracted much attention. Silver is an antibacterial agent, which has antibacterial activity against a variety of drug-resistant strains [20,21]. Zhang et al. demonstrated that AgNPs could promote the proliferation and osteogenic differentiation of MSCs [14]. Hqin et al. found that $4 \mu \mathrm{g} / \mathrm{mL}$ AgNPs (average diameter $19.4 \pm 5.2 \mathrm{~nm}$ ) promoted extracellular matrix mineralization by up-regulating the expression of RUNX2, ALP, BMP-2, COL-1, OPN, and OCN genes, thus promoting the osteogenic differentiation of human urine-derived stem cells [13]. To date, there are few reports on the possible mechanism of the effect of AgNPs on stem cell osteogenesis. AgNPs can induce actin polymerization, increase cytoskeleton tension, activate RhoA, and promote osteogenesis of human umbilical cord blood stem cells [22]. The effect of AgNPs on cell function depends not only on the concentration and incubation time of AgNPs but also on some of their chemical and physical properties, such as their size, shape, surface charge, surface coating or functionalization. The concentrations of AgNPs used in the above studies varied greatly. The physical and chemical properties of silver nanoparticles vary with different AgNP concentrations, which may be the reason for the different results obtained in these studies. In this study, we refer to the results of previous experiments [23].

As the basis of bone tissue engineering, scaffold materials provide an environment for cells to perform biological functions, so biocompatibility is often the primary factor in evaluating the performance of scaffold materials. In this study, CCK-8 results show that Gel/Alg scaffolds containing AgNPs have good biocompatibility. This is not only due to the good biocompatibility of Gel and Alg but also because the scaffolds have appropriate pore sizes and porosities, which provide a large surface area for cell growth, and pore interconnections provide a channel for gas exchange, nutrient transport, and waste discharge. On the first day of the co-culture of MC3T3-E1 cells and scaffold materials, the cells grew well on the surfaces of the scaffold materials. With the increase in time, the proliferation ability of MC3T3-E1 on the surface of scaffolds containing AgNPs strengthened; the proliferation ability of the $200 \mu \mathrm{M}$ AgNP-Gel/Alg scaffolds was the strongest, which may be due to the synergistic effect of AgNPs. Furthermore, the biosafety of three kinds of AgNP-Gel/Alg scaffolds has been proved. Studies have confirmed the dose-dependent cytotoxicity of AgNPs in stem cells. Results from Greulich et al. showed that approximately $100 \mathrm{nM}$ AgNPs at a concentration of more than $5 \mu \mathrm{g} / \mathrm{mL}$ had a clear cytotoxic effect on human bone marrow mesenchymal stem cells, but at a concentration of $2.5 \mu \mathrm{g} / \mathrm{mL}$, they could also induce cell activation and release IL-8 [24]. Qin et al. also demonstrated that low concentrations $(\leq 4 \mu \mathrm{g} / \mathrm{mL})$ of $20 \mathrm{~nm}$ AgNPs did not reduce cell survival, but a high concentration $(4 \mu \mathrm{g} / \mathrm{mL})$ of AgNPs had a significant cytotoxic effect on human USCs [13]. These results suggest that that the AgNPs in this study fall into the range of safe concentration.

In vivo bone defect repair experiments are often used to assess the osteogenic properties of bone tissue engineering materials. Many studies have established animal models of critical bone defects to assess the effect of bone tissue engineering materials on the osteogenesis of bone defects [25-28]. The critical bone defect model of the skull is commonly used at present, which has several advantages compared with models of other parts. First, the skull area has a poor blood supply and weak bone regeneration ability, which is suitable for the study of bone defect repair; second, the operation has a clear field of vision, simplicity, and good repeatability. Finally, in terms of anatomical structure, the dura 
mater can provide support and fixation for bone substitute materials [26]. At present, the reported sizes of critical defects in rabbit skulls vary in the range of 5-15 $\mathrm{mm}$. Jin et al. [27] successfully prepared critical bone defects with an $8 \mathrm{~mm}$ diameter in the rabbit skull and found that the bone defect site contained only a very small amount of new bone formation at 8 weeks. HwKim et al. [28] successfully prepared critical bone defects of $10 \mathrm{~mm}$ and found that there was very little bone formation at 8 weeks. In this study, critical bone defects of $10 \mathrm{~mm}$ in skulls were prepared, and the osteogenic effect of AgNPs was evaluated for 8 weeks.

In this study, Micro-CT and histological analysis of the osteogenic effect of different concentrations of AgNP-Gel/Alg scaffolds were conducted. With the increase in AgNP concentration, the osteogenic effect of the AgNP-Gel/Alg scaffold was not enhanced. The reason may be that AgNPs promote the proliferation and osteogenic differentiation of MSCs, but if the concentration of AgNPs exceeds a certain range, then it may inhibit the activity of these cells. In this study, the Tb.Pf of the $200 \mu \mathrm{M}$ AgNP-Gel/Alg scaffold group was lower than those of other groups $(p<0.05)$. With the increase in time, the Tb.Pf value in the scaffold group decreased accordingly, reflecting the gradual remodeling of immature bone tissue through mineralization and replacement by mature bone trabeculae. Histological analysis showed that there were fewer collagen fibers in the group without AgNPs, while the collagen fibers in the scaffold group containing AgNPs were abundant, and the fibrous tissue was arranged in a regular grid. As time progressed, the fibrous tissue of trabecular bone became thicker and increased. This also suggests that AgNPs can promote the osteogenesis of New Zealand rabbits' skulls.

\section{Conclusions}

- AgNPs have an effect on the physical and chemical properties of Gel/Alg scaffolds, and $200 \mu \mathrm{M}$ AgNP-Gel/Alg scaffolds are more suitable for bone tissue engineering materials.

- AgNP-Gel/Alg scaffolds have good biocompatibility.

- AgNP-Gel/Alg scaffold can promote the repair of skull defects in New Zealand rabbits, and AgNPs have a clear osteogenic effect in vivo.

Author Contributions: All authors contributed equally to this paper and research. Conceptualization, Y.Z. and J.L.; Data curation, B.Z.; Formal analysis, J.H. and M.Z.; Investigation, F.L.; Supervision, Z.Z.; Writing-review and editing, Y.Z. and Y.L. All authors have read and agreed to the published version of the manuscript.

Funding: This research was funded by the Liaoning province key research and development plan project with grant no. (2020JH2/10300038), and Shenyang Science and Technology Project with grant No. (20-205-4-099).

Acknowledgments: The authors would like to express their thanks to teachers and students in the Experimental Centre of School and Hospital of Stomatology, China Medical University, for their continuous help and support.

Conflicts of Interest: The authors declare no conflict of interest.

\section{References}

1. Wang, W.; Yeung, K.W.K. Bone grafts and biomaterials substitutes for bone defect repair: A review. Bioact. Mater. 2017, 2, 224-247. [CrossRef] [PubMed]

2. Winkler, T.; Sass, F.A.; Duda, G.N.; Schmidt-Bleek, K. A review of biomaterials in bone defect healing, remaining shortcomings and future opportunities for bone tissue engineering: The unsolved challenge. Bone Jt. Res. 2018, 7, 232-243. [CrossRef]

3. James, A.W.; Chiang, M.; Asatrian, G.; Shen, J.; Goyal, R.; Chung, C.G.; Chang, L.; Shrestha, S.; Turner, A.S.; Seim, H.B.; et al. Vertebral Implantation of NELL-1 Enhances Bone Formation in an Osteoporotic Sheep Model. Tissue Eng. Part A 2016, 22, 840-849. [CrossRef]

4. Van der, S.J.; Van Lieshout, E.M.; El-Massoudi, Y.; Kralingen, G.H.V.; Patka, P. Bone substitutes in the Netherlands a systematic literature review. Acta Biomater. 2011, 7, 739-750. [CrossRef] [PubMed]

5. Huh, J.; Lee, J.; Kim, W.; Yeo, M.; Kim, G. Preparation and characterization of gelatin/ $\alpha$-TCP/SF biocomposite scaffold for bone tissue regeneration. Int. J. Biol. Macromol. 2018, 110, 488-496. [CrossRef] [PubMed] 
6. Purohit, S.D.; Bhaskar, R.; Singh, H.; Yadav, I.; Gupta, M.K.; Mishra, N.C. Development of a nanocomposite scaffold of gelatin-alginate-graphene oxide for bone tissue engineering. Int. J. Biol. Macromol. 2019, 133, 592-602. [CrossRef] [PubMed]

7. Sun, K.; Li, R.; Jiang, W.; Sun, Y.F.; Li, H. Comparison of three-dimensional printing and vacuum freeze-dried techniques for fabricating composite scaffolds. Biochem. Biophys. Res. Commun. 2016, 477, 1085-1091. [CrossRef]

8. Pinto, J.; Magrì, D.; Valentini, P.; Palazon, F.; Heredia-Guerrero, J.A.; Lauciello, S.; Barroso-Solares, S.; Ceseracciu, L.; Pompa, P.P.; Athanassiou, A.; et al. Antibacterial Melamine Foams Decorated with in Situ Synthesized Silver Nanoparticles. ACS Appl. Mater. Interfaces 2018, 10, 16095-16104. [CrossRef]

9. Abbasi, E.; Milani, M.; Fekri, A.S.; Kouhi, M.; Akbarzadeh, A.; Nasrabadi, H.T.; Nikasa, P.; Joo, S.W.; Hanifehpour, Y.; Nejati-Koshki, K.; et al. Silver nanoparticles, Synthesis methods, bio-applications and properties. Crit. Rev. Microbiol. 2016, 42, 173-180. [CrossRef]

10. Wypij, M.; Jędrzejewski, T.; Ostrowski, M.; Trzcińska, J.; Rai, J.; Golińska, P. Biogenic Silver Nanoparticles: Assessment of Their Cytotoxicity, Genotoxicity and Study of Capping Proteins. Moleciles 2020, $25,3022$. [CrossRef] [PubMed]

11. Liu, X.; Lee, P.Y.; Ho, C.M.; Lui, V.C.H.; Chen, Y.; Che, C.M.; Tam, P.K.H.; Wong, K.K.Y. Silver nanoparticles mediate differential responses in keratinocytes and fibroblasts during skin wound healing. ChemMedChem 2010, 5, 468-475. [CrossRef] [PubMed]

12. Kwan, K.H.; Liu, X.; To, M.K.; Yeung, K.W.K.; Ho, C.H.; Wong, K.K.Y. Modulation of collagen alignment by silver nanoparticles results in better mechanical properties in wound healing. Nanomedicine 2011, 7, 497-504. [CrossRef] [PubMed]

13. Qin,H.; Zhu, C.; An,Z.; Jiang, Y.; Zhao, Y.; Wang, J.; Liu, X.; Hui, B.; Zhang, X.; Wang, Y. Silver nanoparticles promote osteogenic differentiation of human urine-derived stem cells at noncytotoxic concentrations. Int. J. Nanomed. 2014, 9, 2469-2478. [CrossRef] [PubMed]

14. Zhang, R.; Lee, P.; Lui, V.C.; Chen, Y.; Liu, X.; Lok, C.N.; To, M.; Yeung, K.W.K.; Wong, K.K.Y. Silver nanoparticles promote osteogenesis of mesenchymal stem cells and improve bone fracture healing in osteogenesis mechanism mouse model. Nanomedicine 2015, 11, 1949-1959. [CrossRef] [PubMed]

15. Li, X.; Liao, D.; Sun, G.; Chu, H. Odontogenesis and neuronal differentiation characteristics of periodontal ligament stem cells from beagle dog. J. Cell Mol. Med. 2020, 24, 5146-5151. [CrossRef] [PubMed]

16. Flautre, B.; Descamps, M.; Delecourt, C.; Blary, M.C.; Hardouin, P. Porous HA ceramic for bone replacement, role of the pores and interconnections-Experimental study in the rabbit. J. Mater. Sci. Mater. Med. 2001, 12, 679-682. [CrossRef] [PubMed]

17. Kruyt, M.C.; De Bruijn, J.D.; Wilson, C.E.; Oner, F.C.; van Blitterswijk, C.A.; Verbout, A.J.; Dhert, W.J.A. Viable osteogenic cells are obligatory for tissue-engineered ectopic bone formation in goats. Tissue Eng. 2003, 9 , 327-336. [CrossRef]

18. Zhang, Y.; Zhang, M. Three-dimensional macroporous calcium phosphate bioceramics with nested chitosan sponges for load-bearing bone implants. J. Biomed. Mater. Res. 2002, 61, 1-8. [CrossRef]

19. Mao, J.P.; Wei, P.F.; Yuan, Z.Y.; Jing, W.; Cao, J.J.; Li, G.P.; Guo, J.X.; Wang, H.G.; Chen, D.F.; Cai, Q. Osteoconductive and Osteoinductive Biodegradable Microspheres Serving as Injectable Micro-scaffolds for Bone Regeneration. J. Biomater. Sci. Polym. Ed. 2020, 23, 1-15. [CrossRef]

20. Nanda, A.; Saravanan, M. Biosynthesis of silver nanoparticles from Staphylococcus aureus and its antimicrobial activity against MRSA and MRSE. Nanomedicine 2009, 5, 452-456. [CrossRef]

21. Tian, X.; Jiang, X.; Welch, C.; Croley, T.R.; Wong, T.; Chen, C.; Fan, S.; Chong, Y.; Li, R.; Ge, C.; et al. Bactericidal effects of silver nanoparticles on lactobacilli and the underlying mechanism. ACS Appl. Mater. Interfaces 2018, 10, 8443-8450. [CrossRef] [PubMed]

22. Hackenberg, S.; Scherzed, A.; Kessler, M.; Hummel, S.; Technau, A.; Froelich, K.; Ginzkey, C.; Koehler, C.; Hagen, R.; Kleinsasser, N. Silver nanoparticles, Evaluation of DNA damage, toxicity and functional impairment in human mesenchymal stem cells. Toxicol. Lett. 2011, 201, 27-33. [CrossRef] [PubMed]

23. Xu, Y.; Zheng, B.; He, J.; Cui, Z.; Liu, Y. Silver nanoparticles promote osteogenic differentiation of human periodontal ligament fibroblasts by regulating the RhoA-TAZ axis. Cell Biol. Int. 2019, 43, 910-920. [CrossRef] [PubMed] 
24. Greulich, C.; Kittler, S.; Epple, M.; Muhr, G.; Köller, M. Studies on the biocompatibility and the interaction of silver nanoparticles with human mesenchymal stem cells (hMSCs). Langenbecks Arch. Surg. 2009, 394, 495-502. [CrossRef]

25. Hassibi, H.; Farsinejad, A.; Dabiri, S.; Voosough, D.; Mortezaeizadeh, A.; Kheirandish, R.; Azari, O. Allogenic Bone Graft Enriched by Periosteal Stem Cell and Growth Factors for Osteogenesis in Critical Size Bone Defect in Rabbit Model: Histopathological and Radiological Evaluation. Iran. J. Pathol. 2020, 15, 205-216. [CrossRef] [PubMed]

26. Leena, R.S.; Vairamani, M.; Selvamurugan, N. Alginate/Gelatin scaffolds incorporated with Silibinin-loaded Chitosan nanoparticles for bone formation in vitro. Colloids Surf. B Biointerfaces 2017, 158, 308-318. [CrossRef]

27. Shim, J.H.; Moon, T.S.; Yun, M.J.; Jeon, Y.; Jeong, C.; Cho, D.; Huh, J. Stimulation of healing within a rabbit calvarial defect by a PCL/PLGA scaffold blended with TCP using solid freeform fabrication technology. J. Mater. Sci. Mater. Med. 2012, 23, 2993-3002. [CrossRef]

28. Kim, H.W.; Shin, S.Y.; Kim, H.E.; Lee, Y.; Chung, C.; Lee, H.; Rhyu, I. Bone formation on the apatite-coated zirconia porous scaffolds within a rabbit calvarial defect. J. Biomater. Appl. 2008, 22, 485-504. [CrossRef] [PubMed]

(C) 2020 by the authors. Licensee MDPI, Basel, Switzerland. This article is an open access article distributed under the terms and conditions of the Creative Commons Attribution (CC BY) license (http://creativecommons.org/licenses/by/4.0/). 